\title{
Cross reaction of antibodies to a glycine/alanine repeat sequence of Epstein-Barr virus nuclear antigen-1 with collagen, cytokeratin, and actin
}

\author{
C Baboonian, P J W Venables, D G Williams, R O Williams, R N Maini
}

\begin{abstract}
P62 is a synthetic peptide which corresponds to the glycine/alanine repeat sequence of Epstein-Barr virus nuclear antigen-1. It is the main epitope recognised by anti-rheumatoid arthritis nuclear antigen antibodies. It was shown previously that anti-P62 antibodies were raised fourfold in patients with rheumatoid arthritis compared with controls. To examine the possibility that this increase was due to cross reactive autoantibodies binding to $\mathbf{P 6 2}$, anti-P62 antibodies from serum samples taken from 10 patients with rheumatoid arthritis and five healthy controls were purified by affinity chromatography. Immunoglobulin G anti-P62 antibodies purified from four of 10 serum samples from patients with rheumatoid arthritis also reacted with human epidermal keratin, denatured collagen type II and actin, but not with influenza antigens, as determined by enzyme linked immunosorbent assay (ELISA). Anti-P62 antibodies in serum samples from healthy controls and patients with rheumatoid arthritis reacted with epidermal keratin by immunoblotting. It is suggested that antibodies to the glycine/alanine repeat sequence of Epstein-Barr nuclear antigen-1 recognise homologous epitopes on keratin, actin, and collagen. It is also possible that molecular mimicry between a major epitope on the Epstein-Barr virus and several autoantigens might contribute to the breakdown of tolerance and autoimmunity in patients with rheumatoid arthritis.
\end{abstract}

The possibility that the Epstein-Barr virus might have a role in the pathogenesis of rheumatoid arthritis has been suggested by observations that patients with rheumatoid arthritis have high titres of antibodies to an Epstein-Barr virus associated antigen referred to as rheumatoid arthritis nuclear antigen (RANA) (reviewed in ref. 1). Recently we showed that a major epitope for RANA is represented by a synthetic peptide, P62, which corresponds to part of the glycine/alanine internal repeat sequence of the Epstein-Barr nuclear antigen-1 (EBNA-1). ${ }^{2}$ Antibodies to P62 were increased fourfold in patients with rheumatoid arthritis, although levels of antibodies to other components of EBNA-1 were normal. ${ }^{3}$ We suggest that the selectivity of this increased response to the glycine/alanine repeat sequences of EBNA-1 might be related to cross reactions with structural endogenous polypeptides such as cytokeratins and collagens.

In this study we used purified anti-P62 antibodies obtained from serum samples from patients with rheumatoid arthritis and normal healthy controls to study cross reactions with actin, cytokeratins, and collagen using an enzyme linked immunosorbent assay (ELISA) and immunoblotting.

\section{Materials and methods}

Serum samples from 10 patients with classical or definite rheumatoid arthritis ${ }^{4}$ and five healthy volunteers were selected for the presence of high levels of anti-P62 antibodies. Purification of the antibodies was carried out by affinity chomatography as previously described ${ }^{2}$ using cyanogen bromide activated Sepharose (Pharmacia, Hounslow, UK) coupled to $1 \mathrm{mg} / \mathrm{ml}$ of P62 and blocked with ethanolamine. The serum samples $(250 \mu \mathrm{l})$ were applied to $1 \mathrm{ml}$ columns and incubated at room temperature for one hour. After washing with phosphate buffered saline, specifically bound antibody was eluted with $3 \mathrm{M}$ guanidine hydrochloride. The same method was used to purify anti-influenza antibodies, with cyanogen bromide activated Sepharose conjugated to influenza vaccine (Influenza A, Philipine $\mathrm{H} 3 \mathrm{~N} 2$ strain) at a concentration of $5 \mathrm{mg} / \mathrm{ml}$. The eluates were dialysed with phosphate buffered saline and tested by ELISA at the same time as the original serum samples. The serum samples were tested at dilutions of 1:100, 1:500, and 1:2500 in phosphate buffered saline with $0 \cdot 1 \%$ Tween 20 ( $\mathrm{vol} / \mathrm{vol}$ ) and $0.5 \%$ casein (wt/vol) and the eluates at dilutions of $1: 40$ and $1: 100$.

The antigens used were P62, denatured human collagen type II (prepared by limited cold pepsin digestion of human cartilage and purification by differential salt precipitation), ${ }^{5}$ human epidermal keratin (Cambridge BioScience, Cambridge, UK), bovine muscle actin (Sigma Chemical, Poole, UK) and influenza vaccine. The antigens were coated onto the plates at a concentration of $10 \mu \mathrm{g} / \mathrm{ml}$ in phosphate buffered saline. The assays were performed by a conventional ELISA at $37^{\circ} \mathrm{C}$; the anticollagen antibody assay was carried out at $20^{\circ} \mathrm{C}$. Plates coated with casein alone were used as controls. Any reactivity of the serum samples or eluates to casein was subtracted from the absorbance measurements from the antigen plates. A titration curve of absorbance versus serum dilution was plotted for all the serum samples and antigens studied. The ratio of the dilution of the eluate to that of the serum sample that gave the same absorbance value was calculated and expressed as the percentage yield. 
Figure 1

Immunofluorescence microscopy of $H E p-2$ cells. (A) Nucleolar and cytoplasmic fluorescence of a serum sample from a patient with rheumatoid arthritis; (B) anti-P62 antibodies from the same serum sample purified by affinity

chromatography showing cytoplasmic fluorescence only.
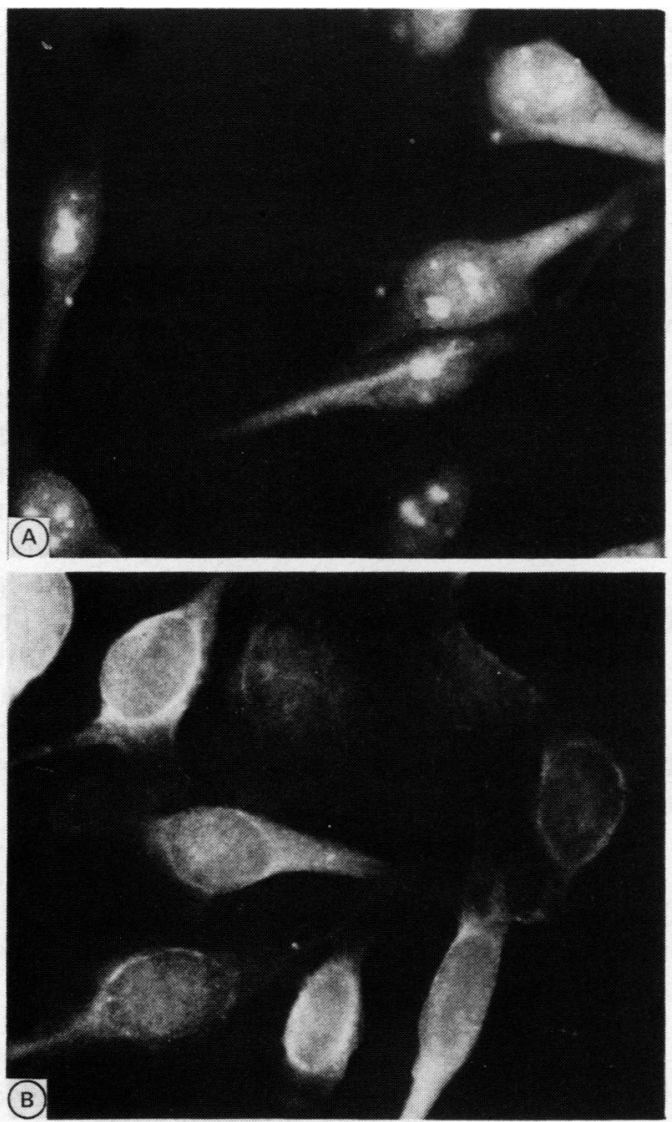

The eluates were also tested for reactivity with EBNA-1 and cross reactivity with human epidermal keratin, actin, and collagen by immunoblotting. Sonicated extracts of EpsteinBarr virus transformed lymphoblastoid cells (Wi-L2 and Raji) and uninfected Ramos cells were loaded into wells of a 5-15\% gradient gel containing about $25 \mu \mathrm{g}$ of total protein per well in addition to $1 \mu \mathrm{g}$ of human epidermal keratin

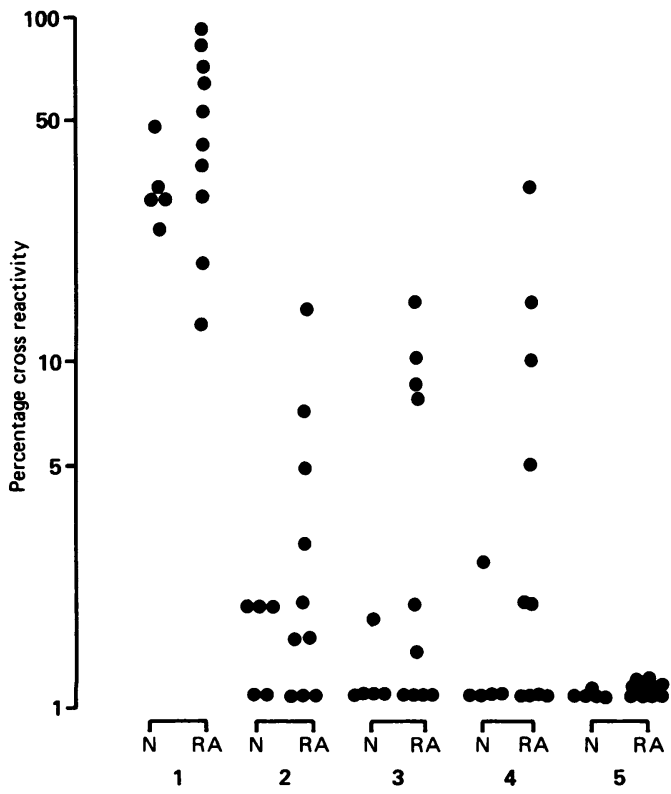

Figure 2 Percentage recovery of purified IgGantibodies to P62 (1) and their cross reaction with denatured collagen (2) human epidermal keratin (3), actin (4), and influenza A(5) from affinity chromatography purification columns. The percentage cross reactivity represents the proportion of the antibody of each specificity which bound to the P62 column The antibodies were purified from serum samples from healthy controls $(N)$ and patients with rheumatoid arthritis $(R A)$. and cyanogen bromide digests of denatured collagen. The transfer to nitrocellulose, the incubation steps with the antibodies purified by affinity chromatography, anti-immunoglobulin-G (IgG) conjugates, and development using a peroxide based ELISA were as reported by Williams et al. ${ }^{6}$ The serum antibodies were examined for antibodies to RANA on B95-8 cells and for antibodies to other cellular antigens on HEp-2 cells.

\section{Results}

On indirect immunofluorescence all 15 preparations of purified anti-P62 antibody from serum samples from controls and patients with rheumatoid arthritis reacted with RANA as previously described. ${ }^{2}$ Four antibody preparations, all from serum samples from patients with rheumatoid arthritis, reacted with cytoskeletal antigens in HEp-2 cells, although none reacted with nuclear antigens in $\mathrm{HEp}-2$ cells. Figure 1 shows that in one serum sample there were antibodies to nucleolar and cytoskeletal antigens, whereas the purified anti-P62 antibody reacted with the cytoskeleton only.

Figure 2 shows that the yield of IgG anti-P62 antibodies (determined by ELISA) purified from normal serum samples was $22-48 \%$ (mean $32 \%$ ), compared with a yield of $13-90 \%$ (mean $50 \%$ ) in patients with rheumatoid arthritis. Anti-P62 antibodies of the IgG class purified from five normal serum samples accounted for less than $2 \%$ of the serum antibody to epidermal keratin, denatured collagen type II, and actin. In contrast, anti-P62 antibodies obtained from four of 10 patients with rheumatoid arthritis cross reacted with all three structural proteins. The level of cross reaction varied between 8.5 and $15 \cdot 4 \%$ for serum antibody to epidermal keratin, between 3.3 and $13.8 \%$ for collagen, and between 4.4 and $33.3 \%$ for actin. No cross

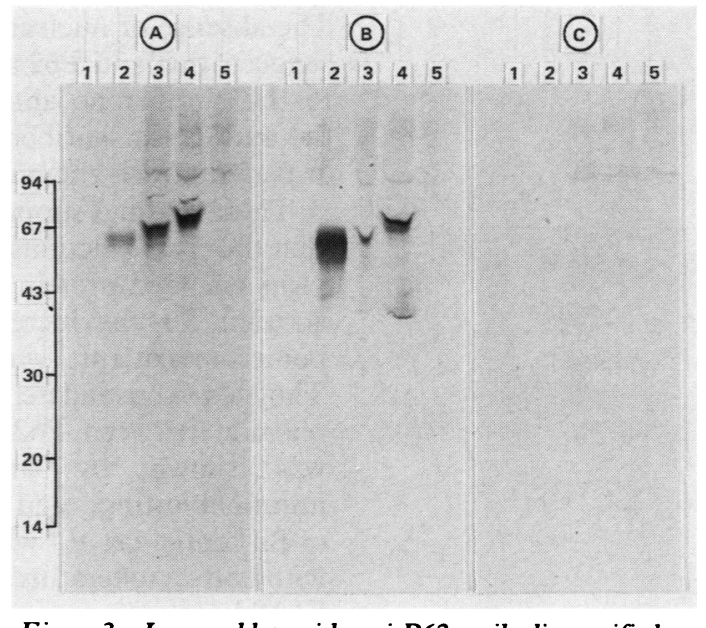

Figure 3 Immunoblots with anti-P62 antibodies purified from serum from a patient with rheumatoid arthritis $(A)$ and from a control serum sample $(B)$. Panel $C$ is an immunoblot with purified anti-influenza antibodies. There was no reaction with collagen polypeptides (lanes 1), but both preparations of anti-P62 antibodies reacted with cytokeratin (lanes 2), the 72 kilodalton Epstein-Barr nuclear antigen-1 (EBNA-1) polypeptide in Raji (lanes 3) and the 80 kilodalton EBNA-1 polypeptide in Wi-L2 (lanes 4). There was background reaction only with EBNA-1 negative Ramos (lanes 5) and with the purified anti-influenza antibodies on all of the antigens. 
reaction with influenza $A$ was observed with anti-P62 antibody purified from any serum sample. As a control for the possibility of nonspecific binding of IgG from some rheumatoid serum samples to affinity chromatography columns, purified antibodies to influenza were prepared from the four serum samples from patients with rheumatoid arthritis, which also contained the highest levels of cross reactive antibody to P62. The reactivity of the four preparations of anti-influenza antibodies with collagen was $1 \cdot 5-3 \%$, with keratin $1 \cdot 5-2 \%$, and with actin $1 \cdot 5-5 \%$ (data not shown).

Figure 3 shows that on immunoblotting, cross reactive anti-P62 antibodies purified from all four serum samples from patients with rheumatoid arthritis reacted with EBNA-1 and with purified epidermal keratin of molecular weight 56 kilodalton. Surprisingly, P62 antibodies from one control serum sample also reacted with keratin by blotting, even though there was no reaction with keratin by ELISA. There was no reaction with purified antiinfluenza antibodies or with the conjugate alone. We were unable to show reactivity with collagen polypeptides nor, in separate experiments, with actin using immunoblotting (data not shown).

\section{Discussion}

This study has shown that serum antibodies to P62, a peptide corresponding to a major epitope of rheumatoid arthritis nuclear antigen, and to the internal repeat sequence of EBNA-1 also react with actin, cytokeratin, and denatured type II collagen, as determined by ELISA. We suggest that these cross reactions may account not only for the increased levels of anti-P62 antibodies in patients with rheumatoid arthritis, but also for some of the autoantibodies which occur in the disease, such as those to actin, cytokeratin, and denatured collagen type II. ${ }^{1}$ The absence of nuclear staining on HEp-2 cells suggests that anti-P62 antibodies are not related to the antihistone antibodies which represent the antinuclear antibodies found in about $40 \%$ of patients with rheumatoid arthritis. ${ }^{7}$

These findings support our original hypothesis that the cross reactions between an Epstein-Barr virus encoded protein and a host protein could account for the increase of anti-RANA antibodies in patients with rheumatoid arthritis. ${ }^{8}$ They also consolidate our suggestion of a cross reaction between $\mathrm{P} 62$ and cytokeratin, which was shown by immunofluorescence and immunoblotting, ${ }^{3}$ and also confirm the findings of Birkenfield et al, ${ }^{9}$ who showed cross reactions with both cytokeratins and collagen by inhibition ELISA.

The design of our study has excluded several possible non-specific interactions between immunoglobulins and certain antigens as possible contributions to the cross reactions. $\mathrm{P} 62$, which is entirely composed of the neutral amino acids glycine and alanine, is unlikely to take part in non-specific charge-charge interactions. Evidence against the participation of the binding of the Fc component of IgG to the column or peptide, or other non-specific interactions, was provided by the absence of any anti-influenza or antinuclear antibody activity of the purified anti-P62 antibodies and the much lower levels of apparent cross reactivity of the purified anti-influenza antibodies. The same experiments confirm that our findings cannot be attributed to the theoretical possibility that the anti-P62 antibodies were copurified with rheumatoid factors, which in turn were bound to other IgG molecules with different specificities.

The cross reaction of anti-P62 antibodies with cytokeratin was confirmed by immunoblotting. The absence of binding to collagen or actin in the same experiments is unexplained, although it is possible that the epitopes seen on ELISA in the native molecules or after gentle denaturation at $56^{\circ} \mathrm{C}$ did not survive boiling, reduction, or treatment with sodium dodecyl sulphate. It was also noteworthy that the P62 antibodies did not bind to polypeptides other than EBNA-1 in the cell extracts. Rhodes et $a l^{10}$ showed that the cross reactivity of $\mathrm{P} 62$ antibodies in infectious mononucleosis serum samples was more extensive in that the antibodies reacted with several bands on immunoblotting. This finding is probably attributable to the class (IgM rather than IgG) of P62 antibodies in this disease. Among the many reactive autoantigens, Rhodes et $a l^{10}$ also found evidence of cross reactivity with cytokeratin.

The molecular basis of these cross reactions is probably reflected in the structure of $\mathrm{P} 62$ and the internal repeat sequence (IR3) of EBNA-1 from which it is derived. The IR3 region is entirely composed of the amino acids glycine and alanine with P62, having the sequence AGAGGGAGGAGAGGGAGGAGA. The high concentration of glycine ( $65 \%$ of the peptide) may explain the reaction of anti-P62 and antiEBNA-1 with Sepharose conjugated to glycine observed previously. ${ }^{2}$ It may also account for the cross reaction with cytokeratins which contain long polyglycine sequences in addition to some glycine/alanine and glycine/serine repeats. ${ }^{11}$ Allowing for conservative substitutions, such as alanine/serine, type II collagen also shows homologies with P62 of up to six of eight amino acids (reviewed in ref. 12). Actin shows fewer similarities, although the sequence from position 11-18 GSGLCKAGF of human smooth muscle actin is identical in four of eight amino acids. ${ }^{13}$ An additional factor which must be considered in apparent cross reactions with actin is that this protein is notoriously 'sticky' in antibody assays and is often identified as an extra polypeptide in immunoprecipitation systems. ${ }^{14}$ The possibility that such an interaction occurred in the ELISA in our study is suggested by the finding that antibodies purified on the control (influenza) column showed the greatest reaction with actin-up to $5 \%$ of the original serum antibody. The fact that the binding of purified anti-P62 antibodies to actin was considerably higher (up to $33 \%$ ) suggests that there was an additional binding component owing to a true cross reaction between actin and P62.

These data suggest that the autoimmune response in patients with rheumatoid arthritis 
may not be as heterogeneous as was originally thought. Possibly, anti-RANA, and some of the anticytokeratin, anticollagen and antiactin antibodies are, if not the same, closely related to each other by their reactivity with glycine rich repeat sequences. Whether the Epstein-Barr virus plays a part in the generation of such antibodies is still unknown, although it is possible that the presence of such a sequence in an immunogenic part of one of its major antigens might contribute to the breakdown of tolerance with the subsequent generation of antibodies to structural proteins. Such antibodies, particularly those against collagen, could play a role in the pathogenesis of rheumatoid arthritis.

The authors thank Dr Richard Smith, Johnson, and Johnson, CA, USA, for the generous gift of the P62 peptide. This project was supported by grants from the Arthritis and Rheumatism Council of Great Britain and the Oliver Bird Fund, Nuffield Foundation for Medical Research.

1 Venables P J W. Epstein-Barr virus infection and autommunity in rheumatoid arthritis [leading article]. $A n n$ Rheum Dis 1988; 47: 265-9.

2 Venables P J W, Pawlowski T, Mumford P A, Brown C, Crawford D H, Maini R N. Reaction of antibodies to rheumatoid arthritis nuclear antigen with a synthetic peptide corresponding to part of Epstein-Barr nuclear antigen-1. Ann Rheum Dis 1988; 47: 270-9.

3 Baboonian C, Halliday D, Venables P J W, Pawlowski T,
Millman G, Maini R N. Antibodies in rheumatoid arthritis react specifically with the glycine alanine repeat sequence of Epstein-Barr nuclear antigen-1. Rheumatol Int 1989; 9; 161-6.

4 Ropes M W, Bennet G A, Cobb S, Jacox R, Jessar R A. Revision of criteria for rheumatoid arthritis. Bull Rheum Dis 1959; 9: 175-6.

5 Rowley M, Tait B, Mackay I R, Cunningham T, Philips B. Collagen antibodies in rheumatoid arthritis. Significance of antibodies to denatured collagen and their association with HLA-DR4. Arthritis R heum 1986; 29: 174.

6 Williams D G, Stocks M R, Charles P J, Maini R N. Antibodies to $\mathrm{La}, \mathrm{Jo}-1, \mathrm{nRNP}$ and $\mathrm{Sm}$ detected by a multitrack immunoblotting using a novel filter holder. A comparative study with counterimmunoelectrophoresis using sera from patients with systemic lupus erythematosus and Siogren's syndrome. F Immunol Methods 1986; 91: 65-73.

7 Tan E M. Autoantibodies to nuclear antigens (ANA): their immunobiology and medicine. In: Kunkel H G, Dixon F J, eds. Advances in immunology. Vol 33. New York: Academic eds. Advances in imming

8 Venables P J W, Roffe L M, Erhardt C C, Maini R N, Edwards J M B, Porter A D. Titers of antibodies to RANA in rheumatoid arthritis and normal sera. Arthritis Rheum 1981; 24: 1459-64.

9 Birkenfield P, Haratz N, Klein G., Cross-reactivity between the EBNA-1 p107 peptide, collagen and keratin: implications for the pathogenesis of rheumatoid arthritis. Clin Immunol Immunopathol 1981; 54: 14-25.

10 Rhodes G, Rumpold R, Kurki P, Patrick K M, Carson D A Vaughan $J \mathrm{H}$. Autoantibodies in infectious mononucleosis have specificity for the olycine alanine repeating region of the Epstein-Barr nuclear antigen. $\mathcal{f} \operatorname{Exp} M e d$ 1987; 165: 1026-40.

11 Fuchs E Hanukoglu I. Unravelling the structure of the intermediate filaments. Cell 1983; 34: 332-4.

12 Venables $P$ J W. Infections and rheumatoid arthritis. Cur Opin Rheumatol 1989; 1: 15-20.
Oenables P J W. Infections and

13 Ueyama H, Hamada H, Battula N, Kakunaga T. Structure of a human smooth muscle actin gene (aortic type) with a unique intron site. Mol Cell Biol 1984; 4: 1073-8.

14 Mathews M B, Bernstein R M. Myositis autoantibody inhibits histidyl-tRNA synthetase: a model for autoimmunity. Nature 1983; 304: 177-9. 